\title{
Regions of the MPFC differentially tuned to social and nonsocial affective evaluation
}

\author{
Lasana T. Harris and Samuel M. McClure \\ Princeton University, Princeton, New Jersey \\ WOUTER VAN DEN BOS \\ University of Amsterdam, Amsterdam, The Netherlands \\ Jonathan D. COHEN \\ Princeton University, Princeton, New Jersey \\ and University of Pittsburgh Medical School, Pittsburgh, Pennsylvania \\ AND \\ SUSAN T. FISKE \\ Princeton University, Princeton, New Jersey
}

\begin{abstract}
The medial prefrontal cortex (MPFC) reliably activates in social cognition and reward tasks. This study locates distinct areas for each. Participants made evaluative (positive/negative) or social (person/not a person) judgments of pictured positive or negative people and objects in a slow event-related design. Activity in an anterior rostral region (arMPFC) was significantly greater for positive than for negative persons but did not show a valence effect for objects, and this was true regardless of the judgment task. This suggests that the arMPFC is tuned to social valence. Interestingly, however, no regions of the MPFC were found to be responsive to social information independently of valence. A region-of-interest analysis of the paraanterior cingulate cortex (pACC), previously implicated in reward processing, demonstrated sensitivity to the valence of all stimuli, whether persons or objects, across tasks. Affective evaluation may be a general function of the MPFC, with some regions being tuned to more specific domains of information (e.g., social) than are others.
\end{abstract}

A region of the prefrontal cortex, the medial prefrontal cortex (MPFC) has been implicated in a wide variety of tasks involving social cognition, as well as reward (Amodio \& Frith, 2006). This dual pattern of findings raises questions about the specific functions served by regions within the MPFC.

Social neuroscience findings have suggested that the MPFC is a necessary component of social cognition. The para-anterior cingulate cortex (pACC) specifically, a region within the MPFC just anterior to the cingulate, is included in a face perception network (Haxby, Gobbini, \& Montgomery, 2004), along with other areas, such as the amygdala, insula, superior temporal sulcus (STS), fusiform gyrus of the temporal cortex, precuneus, and posterior cingulate. Theory of mind (ToM) studies (Castelli, Happé, Frith, \& Frith, 2000; Fletcher et al., 1995; C. D. Frith \& U. Frith, 2006; U. Frith \& C. Frith, 2001; Gallagher \& Frith, 2002; Grèzes, Frith, \& Passingham, 2004a, 2004b; Rilling, Sanfey, Aronson, Nystrom, \& Cohen, 2004; Saxe, Carey, \& Kanwisher, 2004; Saxe \& Wexler, 2005) and studies of dispositional attribution (Harris,
Todorov, \& Fiske, 2005) also have included areas of the MPFC as part of a social cognition network.

In contrast, a growing number of neuroimaging studies examining decision making and evaluation processes have also consistently implicated the MPFC - and specifically, the pACC - as a component of a reward-processing network that also includes the anterior and posterior cingulate cortices and the ventral striatum (Knutson, Fong, Bennett, Adams, \& Hommer, 2003; McCabe, Houser, Ryan, Smith, \& Trouard, 2001; McClure, Laibson, Loewenstein, \& Cohen, 2004; Montague, King-Casas, \& Cohen, 2006; Rilling et al., 2002; Schultz, Dayan, \& Montague, 1997). This reward network is responsive in tasks that often do not involve explicit social thinking. For instance, decisions for and receipt of monetary and food rewards are not traditionally social stimuli and yet often activate the pACC (Berns, McClure, Pagnoni, \& Montague, 2001; Daw, O'Doherty, Dayan, Seymour, \& Dolan, 2006; Delgado, Miller, Inati, \& Phelps, 2005; Hampton \& O'Doherty, 2007; Knutson, Taylor, Kaufman, Peterson, \& Glover, 2005; McClure, Ericson, Laibson, Loewenstein, \& Cohen, 2007; McClure

L.T. Harris, lasana@nyu.edu 
et al., 2004). One might argue that rewards are often socially determined (money is an arbitrary symbol; foods are culturally determined as pleasant or not). And rewards are often conveyed socially - that is, delivered by other people. However, it seems unlikely that responses to the automated delivery of primary rewards (such as juice) in simple conditioning tasks reflect social, rather than more basic, evaluative processes.

How then to interpret the persistent activation of the MPFC in social, as compared with nonsocial, conditions in such a wide array of tasks? Perhaps reward circuitry, including regions within the MPFC, responds to social stimuli because most carry intrinsic reward. Indeed, the social cognition literature strongly suggests this; most people perceive themselves and others as positive by default (Fiske, 2004, pp. 23-24; Kwan, John, Kenny, Bond, \& Robins, 2004; Matlin \& Stang, 1978; Sears, 1983; Taylor \& Brown, 1988). Of course, not everyone is perceived positively. But consistent with the link of the MPFC to positive social stimuli, the most extremely negative outgroups actually reduce MPFC activity (Harris \& Fiske, 2006). Nevertheless, positive expectations are clearly the typical baseline. For example, people evaluating most other people will use only the top half of a rating scale. In social contexts, negative people and behavior stand out and receive disproportionate attention and weight (Fiske, 1980). Thus, social stimuli may be a pervasive type of positive reward, activating a key component of the reward circuitry including regions within the MPFC.

Another explanation for responses in the MPFC to both social and reward-related stimuli may be that different regions within the MPFC participate in distinct circuits that serve different types of evaluative processing, some specifically social and some broader. Indeed, social manipulations typically do not engage other reward-related areas, such as the ventral striatum, and reward manipulations do not reliably engage other socially relevant areas, such as the amygdala, STS, fusiform temporal gyrus, and precuneus. Thus, social and broader evaluative circuits may engage neighboring but distinct regions within the MPFC.

A prior social neuroscience study has provided evidence for positive social affect as activating the MPFC, and it motivated our paradigm here. Social groups systematically differentiate along two primary and universal dimensions of person perception (warmth and competence; Fiske, Cuddy, \& Glick, 2007). The most extremely negative groups (e.g., homeless people), low on both dimensions, generate no positive affect but, instead, the basic negative emotional reaction of disgust (Fiske, Cuddy, Glick, \& Xu, 2002). As has been noted, these extremely negative social groups also do not generate MPFC activity significantly above a fixation baseline, unlike all other social groups. Activation of regions of the MPFC even for groups that have mixed (some negative, some positive) aspects is equivalent to that for the most positive groups (e.g., ingroups). The low-low outgroups elicit dramatically reduced MPFC activity in this region, as compared with all social groups that are positive on at least one dimension (the other groups also generate exclusively social emotions: either ambivalence [envy and pity] or outright positivity [pride]; Harris \& Fiske, 2006). Social stimuli carry valence, and positive social stimuli engage evaluative mechanisms associated with positive affect under other conditions (Fiske \& Taylor, 2008).

On the other hand, another social neuroscience study has strongly argued for the MPFC's role in social perception, even when affect is less obviously involved. Participants were asked to attribute behavior to a target person's disposition, to some situational variable, or to a combination of factors (Harris et al., 2005). This study, an imaging replication of an established social psychological paradigm (see Kelley, 1969; McArthur, 1972), presented sentences describing negative, positive, and neutral behaviors of a target. Additional information next specified the behavior's social conformity, its consistency, and its target's distinctiveness. The combination of information that led to a dispositional attribution about the person performing the behavior (low conformity, high consistency, or low distinctiveness) also increased MPFC activity in a region superior to the $\mathrm{pACC}$, whereas the combination of information that led to a situational or complex attribution decreased activity in this region of the MPFC. Dispositional information also activated other ToM neural areas. In addition, a replication of this paradigm, using objects instead of people, did not show MPFC activation for dispositional information, although the behavioral result was comparable (Harris \& Fiske, in press). Thus, evidence from this and other studies of ToM suggest that at least some regions of the MPFC are responsive to social information independently of evaluation. Thus, the question remains whether regions of the MPFC engaged in social tasks reflect functions involved in the processing of social information as such or a more general role of the MPFC in evaluation that includes social information.

\section{Regional Distinctions Within the MPFC}

The foregoing considerations suggest that the MPFC may comprise subregions that code different types of information. Amodio and Frith (2006) have suggested a scheme for dividing the MPFC into subregions that may be relevant to such functional distinctions. In the present article, we will use the Amodio and Frith scheme, with an important additional distinction. Accordingly, we distinguish between three regions of the MPFC: the posterior rostral medial prefrontal cortex (prMPFC), the anterior rostral medial prefrontal cortex (arMPFC), and the orbital medial prefrontal cortex (oMPFC). The prMPFC and arMPFC share an oblique boundary at Talairach $y=15$, $z=15$ to $y=45, z=45$, whereas the arMPFC and oMPFC share the Talairach boundary $z=2$.

In addition, we identify the pACC as a distinct region within the MPFC. It is subsumed as part of the arMPFC, using the Amodio and Frith (2006) distinctions, but is distinguished from more superior and anterior parts of the arMPFC. We make this additional distinction because of findings in the literature identifying the $\mathrm{pACC}$ as active in both social cognition and reward paradigms. The location of this pACC region of interest (ROI) is based on previous studies implicating this region in reward processing (Knutson, Adams, Fong, \& Hommer, 2001; McClure et al., 
2004; Nieuwenhuis et al., 2005; O’Doherty, Kringelbach, Rolls, Hornak, \& Andrews, 2001; O’Doherty et al., 2003) and social cognition (Gallagher \& Frith, 2002, McCabe et al., 2001; Rilling et al., 2004; Sanfey, Rilling, Aronson, Nystrom, \& Cohen, 2003). The ROI consists of 33 voxels in a sphere centered at Talairach coordinates $x=0, y=$ $44, z=12$.

\section{Evaluative and Social Cognition Predictions}

The aim of the experiment was to dissociate social and evaluative processes in the MPFC. We hypothesize that the MPFC comprises distinct regions that respond differently to social and nonsocial valenced information. The pACC should distinguish between positive and negative valence irrespective of social content, consistent with the role of the pACC in reward processing noted above. However, because people are a common source of positive affect, as has been noted, we predicted a greater attunement to people's reward value and, therefore, a greater differentiation of positive-negative valence for people, rather than objects, in other areas of the MPFC. We also predicted that regions of the MPFC superior to the pACC would differentiate the valence of only social stimuli, consistent with the social neuroscience literature (see Harris et al., 2005). Finally, we examined the MPFC in two separate tasks, a person perception task and an evaluative one, to assess potential differences due to task context.

\section{METHOD}

\section{Participants}

Twelve participants completed the imaging study for $\$ 30$. All the participants were undergraduate students at Princeton University The mean age for the sample was 19.5 years, with 7 females and 4 students of Black, Asian, or mixed descent. All the participants were right-handed and had no history of neurological disorders or brain trauma.

\section{Stimuli}

The task involved looking at positive and negative pictures of persons and objects. Pictures of objects were taken from the International Affective Picture System (IAPS; Lang \& Bradley, 1994). An equivalent number of positive and negative pictures of objects were selected from the 40 highest valence and lowest valence pictures. The 30 selected pictures received the highest and lowest ratings on this bipolar valence dimension (see Lang \& Bradley, 1994). Pictures were omitted from this group if they contained people or repeated a previous object from another angle.

Pictures of persons were taken from an unpublished social groups picture set used previously by two of the authors (see Harris \& Fiske, 2006). Each photograph depicted an individual easily identified as belonging to a social category pretested for valence. Those pictures that elicit the basic negative emotion (disgust) were characterized as negative person pictures throughout this study. Other social group pictures are relatively more positive (they elicit at least partially positive social emotions of pride, envy, and pity) and are characterized as positive person pictures. ${ }^{1}$ All the pictures were otherwise randomly chosen from the larger database.

Stimuli varied in target size and picture quality, but these differences were evenly distributed across the positive-negative and person-object distinctions.

\section{Scanning Parameters}

All fMRI scanning was conducted at Princeton's Center for the Study of Brain, Mind, and Behavior, using a 3.0-tesla Siemens Al- legra head-dedicated MR scanner. A Dell computer projected images onto a screen mounted at the rear of the scanner bore, which the participants viewed, while supine, through a series of mirrors. Prior to the functional echo planar image (EPI) acquisitions, the subjects received a short series of structural MRI scans to allow for subsequent functional localization. These scans took approximately 12 min and included (1) a brief scout for landmarking and (2) a highresolution $(0.5 \times 0.5 \times 1.0 \mathrm{~mm}) \mathrm{T} 1$-weighted anatomical image whole-brain MPRAGE sequence for later localization and intersubject registration. Functional imaging then proceeded using an EPI sequence that allowed for whole-brain coverage (thirty-two 3-mm axial slices; 1-mm gap; TR, 2 sec; TE, $30 \mathrm{msec}$ ). In-plane resolution was $3 \times 3 \mathrm{~mm}(196-\mathrm{mm}$ FOV, $64 \times 64$ matrix $)$.

\section{Procedure}

The participants were instructed before scanning that they would lie in the scanner while pictures of different people and objects appeared on the screen. Their task was simply to look at the pictures and make simple judgments (described below).

Pictures appeared on the screen for $500 \mathrm{msec}$. The short display time was chosen to prevent the participants from looking away from the negative pictures. A red fixation cross separated each picture and remained on the screen for $11 \mathrm{sec}$. One second before a picture appeared, the fixation cross would turn from red to green. The green fixation cross cued the participant, and a picture appeared.

The participants performed one of two tasks while looking at the pictures. In the social judgment task, the participants indicated whether the picture was a person or not a person. In the evaluative judgment task, the participants indicated whether the picture was positive or negative. Tasks appeared in a separate random sequence for each participant. They were encouraged to respond as quickly as possible after each stimulus presentation. The participants responded by pressing the corresponding key on a button box in their right hand. A cue screen at the beginning of each task assigned keys to their corresponding responses. Assignment of options to index and middle buttons was counterbalanced across tasks.

A run consisted of both tasks, counterbalanced across participants. A cue screen at the beginning of the task signaled the current task to the participant. Twelve stimuli were then presented, randomly selected from the pool of 60 . At the midpoint of each run, a second cue screen appeared, signaling to the participant that the second task was about to begin and once again assigning the keys to the corresponding responses. Twelve more pictures, randomly selected with replacement, then appeared. Each participant completed five runs, viewing and responding to each picture once in each task. Pictures appeared at most once in each task and each separate run.

\section{Data Analysis}

Data preprocessing and statistical analysis used AFNI (Cox, 1996). Preprocessing consisted of slice time correction using the Fourier method, rigid-body 3-D motion correction using Fourier interpolation, and spatial smoothing with a 6-mm full-width half maximum Gaussian kernel. The functional data were then aligned with the anatomical data. First, the functional data were rotated to the same angle as the anatomical data before the functional data were shifted in three dimensions to find the best fit. Spatial normalization was done using the automatic AFNI program @auto_tlrc, which normalized the images and data to Talairach space (Talairach \& Tournoux, 1988).

Statistical analyses were performed using a general linear model (GLM) in which the event-related design was modeled using a canonical hemodynamic response function. The GLM consisted of 14 regressors: 2 tasks (social or evaluative) $\times 2$ types of stimuli (people or objects) $\times 2$ valence (positive or negative), plus 6 regressors estimating the movement history. This analysis was performed on each participant's data set individually. The contrast between parameter estimates for different events within each participant were submitted to a group analysis (ANOVA) that treated the variability between participants as a random effect. Statistical parametric maps were derived from the resulting $t$ values associated with each voxel. 
A
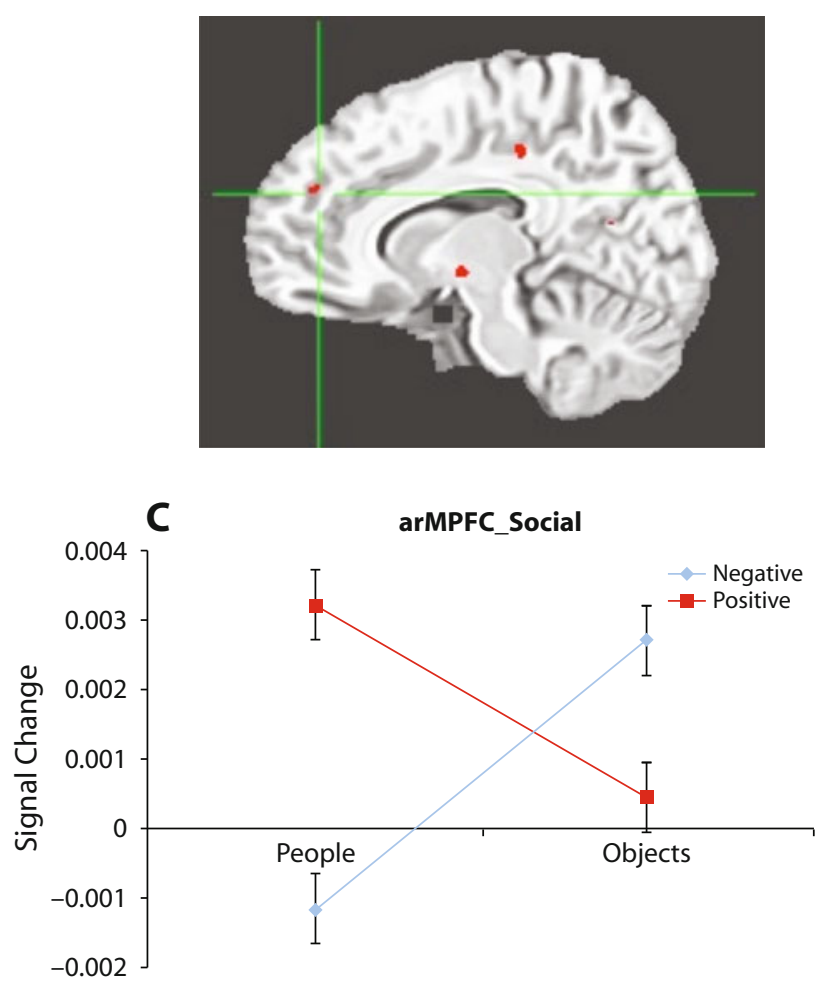
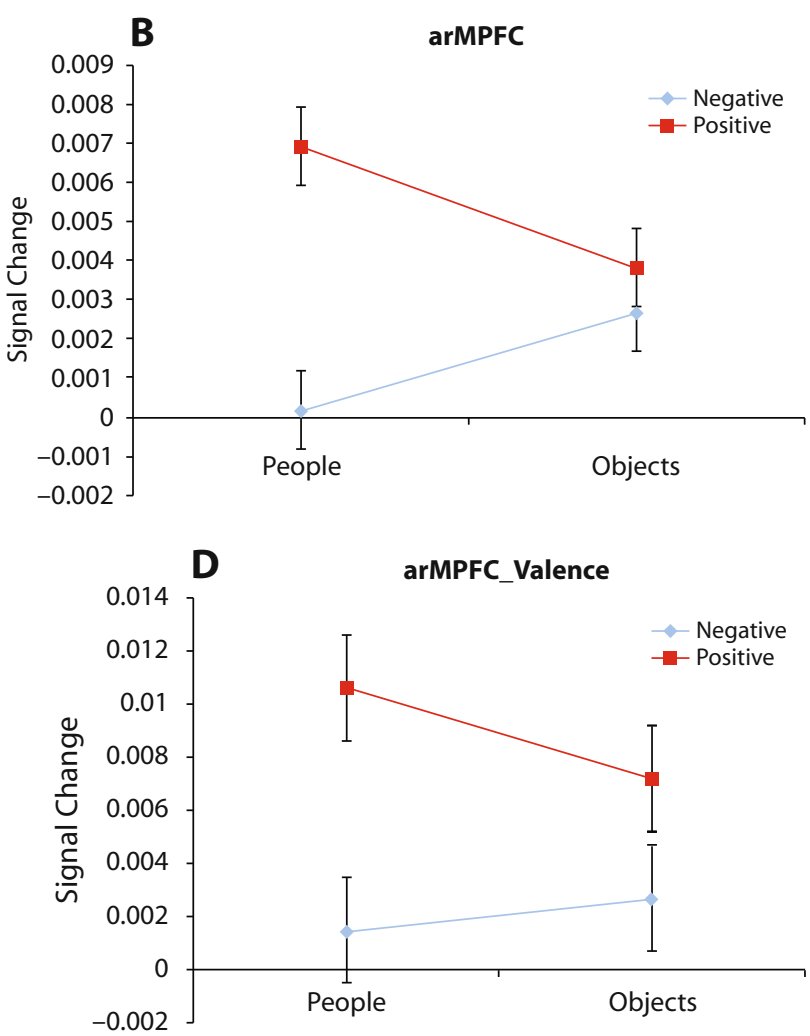

Figure 1. Area of the anterior rostral medial prefrontal cortex (arMPFC) that shows greater activation to positive than to negative persons. Line graphs correspond to activity in the cluster. (A) Cluster of the arMPFC, located in crosshairs. (B) Main effect. (C) Within social task. (D) Within valence task.

All reported statistics are significant at $p<.01$. Reported $p$ values represent the mean $p$ value of the voxels in the designated cluster. Only clusters with 10 or more contiguous voxels exhibiting activity significant above the $p<.01$ level were included. This combination of voxel-wise $p$ value and extent thresholding reduced the effective per voxels false positive rate to family-wise $\alpha=.01$ (Forman et al., 1995). The locations of peak activity of the reported clusters are in Talairach coordinates.

We first performed whole-brain analyses designed to identify regions responsive to socially tuned comparisons: differences between positive and negative persons that are greater than those for objects and differences between persons and objects. We then performed an ROI analysis of the pACC. This analysis was guided by previous findings in the literature and allowed us to compare activity in this specific area of MPFC with those identified in the whole-brain analyses as socially tuned. By so doing, we could determine whether this region was sensitive to evaluative information (e.g., positive vs. negative stimuli) irrespective of social information and, thereby, could test the hypothesis that different regions of the MPFC respond to social versus nonsocial evaluative information.

\section{RESULTS}

\section{Whole-Brain Analyses}

We will report only significant results from the threeway valence $\times$ stimulus type $\times$ task interaction. Lower order interactions and simple effects from nonsignificant three-way interactions will not be reported unless motivated by our a priori hypotheses.
Stimulus $\times$ valence interaction. If some regions of the MPFC selectively respond to social evaluative information, these should show significantly more activity to positive than to negative persons, but less or no difference for objects, across tasks. To assess this, we tested for a stimulus $\times$ valence interaction - that is, the effect of valence compared within persons and objects. One area of the arMPFC was more active to positive people than to negative people $[t(11)=3.02, p<.01$, at $x=11, y=45$, $z=27$; 11 voxels; see Figure 1A] but did not show this valence effect for objects $[t(11)=0.44, p=.67, \mathrm{n} . \mathrm{s}$. $]$. This suggests that at least one area of the MPFC is sensitive to the qualitative difference between target people who elicit more basic negative emotions and those who elicit more complex positive social emotions (see Table 1 for other active neural regions for this contrast). In contrast, no area of the MPFC was selectively sensitive to the difference between positive and negative objects, although other brain regions beyond the MPFC did show this form of the stimulus $\times$ valence interaction (see Table 2 ). In addition, the activation to positive persons was significantly greater than the activity for both positive and negative objects, whereas the activity for negative persons was significantly less than that for negative objects across tasks.

Within task, both the person judgment task and the evaluative task revealed a similar pattern: Activity in the arMPFC was greater to positive persons than to negative 
Table 1

Brainwide Regions Significant for the Stimulus $\times$ Valence Interaction, in Which There Is a Significant Valence Effect of Positive > Negative, but Only for People, Not Objects

\begin{tabular}{lrrrr}
\hline \multicolumn{1}{c}{ Brain Region } & \multicolumn{1}{c}{$x$} & \multicolumn{1}{c}{$y$} & \multicolumn{1}{c}{$z$} & Cluster Size \\
\hline Right posterior cingulate & -5 & -63 & 11 & 381 \\
Right putamen & 24 & -5 & -5 & 206 \\
Right inferior parietal lobule (BA 40) & 65 & -35 & 34 & 162 \\
Left inferior frontal gyrus (BA 9) & 41 & 6 & 27 & 116 \\
Left posterior cingulate & 17 & -53 & 13 & 85 \\
Left middle frontal gyrus & 43 & 26 & -34 & 83 \\
Right middle temporal gyrus (BA 37) & -49 & -65 & 7 & 83 \\
Right precuneus (BA 7) & -5 & -32 & 43 & 64 \\
Right supramarginal gyrus (BA 40) & -42 & -40 & 35 & 62 \\
Left subthalamic nucleus & 11 & -10 & -2 & 45 \\
Left cingulate (BA 31) & 9 & -27 & 42 & 39 \\
Right cingulate (BA 24) & -8 & 11 & 32 & 26 \\
Right hippocampus and & & & & \\
$\quad$ parahippocampal gyrus & -33 & -17 & -15 & 26 \\
Left middle frontal gyrus (BA 6) & 27 & -9 & 55 & 19 \\
Right superior temporal sulcus & -52 & -30 & 13 & 13 \\
Left anterior rostral medial & & & & \\
$\quad$ prefrontal gyrus (BA 9) & 11 & 45 & 27 & 11 \\
Left middle temporal gyrus & 37 & -65 & 29 & 11 \\
\hline
\end{tabular}

Note-All reported statistics are significant at $p<.01$, and all reported clusters contain at least 10 contiguous voxels. There were no regions significantly more active for the opposite comparison.

persons, and the difference between positive and negative stimuli was larger for persons than for objects (see Figures $1 \mathrm{C}$ and $1 \mathrm{D}$ ). This replicates the social-tuning finding across tasks, in both social and evaluative contexts.

Stimulus factor main effect. To determine whether there are areas of the MPFC tuned to social information irrespective of evaluation, we tested for a person-object main effect (i.e., collapsed across stimuli and tasks). No area showed this main effect. Areas beyond the MPFC were more active for objects than for persons (see Table 3). This suggests that no area within the MPFC was particularly selective to social information independently of evaluation. ${ }^{2}$

\section{Para-Anterior Cingulate ROI Analysis}

We examined a cluster of 33 voxels directly anterior to the horn of the cingulate to determine whether the area referred to as the pACC in neuroeconomic studies discriminated between persons and objects of positive and negative valence (Figure 2A). ${ }^{3}$ We did not observe a significant stimulus $\times$ valence interaction in this ROI, nor was the three-way interaction with task significant. There was, however, a signifi-

Table 2

Brainwide Regions Significant for the Stimulus $\times$ Valence Interaction, in Which There Is a Significant Valence Effect, but Only for Objects, Not People

\begin{tabular}{|c|c|c|c|c|}
\hline Brain Region & $x$ & $y$ & $z$ & Cluster Size \\
\hline \multicolumn{5}{|c|}{ Positive $>$ Negative } \\
\hline Right precentral gyrus & -62 & 4 & 21 & 120 \\
\hline Left middle temporal gyrus & 53 & -67 & 28 & 62 \\
\hline Right middle temporal gyrus & -48 & -25 & -6 & 29 \\
\hline \multicolumn{5}{|c|}{ Negative $>$ Positive } \\
\hline Right superior temporal sulcus & -61 & -58 & 10 & 52 \\
\hline
\end{tabular}

Note-All reported statistics are significant at $p<.01$, and all reported clusters contain at least 10 contiguous voxels. cant valence main effect, so that, across tasks and stimuli, positive stimuli elicited a stronger response than did negative stimuli (Figure 2B). There was no stimulus or task main effect. Similarly, in the evaluative task, the pACC was significantly more active to positive than to negative valence for both persons and objects (see Figure 2D); the valence difference for each stimulus type did not differ. ${ }^{4}$

\section{DISCUSSION}

An area of the arMPFC showed greater activity to positive persons than to negative persons but no such difference for positive and negative objects across both an evaluative and a social task. This region of the MPFC seemed to be socially tuned: It differentiated valence mainly of social stimuli, regardless of the participant's judgment task. At the same time, it seems to have been specifically tuned to valence (i.e., evaluative) information, inasmuch as it did not show activity in response to social versus nonsocial information when this was collapsed across valence.

Distinct from the arMPFC, the pACC showed a response to valence irrespective of stimulus type or task. This is consistent with findings from previous studies of reward and economic decision making, suggesting that this area is responsive to evaluative information across a broad range of domains.

Taken together, our findings suggest that different regions within the MPFC may be differentially tuned to different types of evaluative information, with the arMPFC responding specifically to valence differences in social, rather than nonsocial, stimuli and the pACC responsive to a broader general class of evaluative information. These findings suggest that affective evaluation may be a general function of the MPFC, with some areas showing greater domain specificity than do others. 
Table 3

Brain Areas Activated More to Objects Than to Persons

\begin{tabular}{lcccc}
\hline \multicolumn{1}{c}{ Brain Region } & $x$ & $y$ & $z$ & Cluster Size \\
\hline Right precentral gyrus & -36 & -9 & 27 & 1,907 \\
Left cingulate & -12 & -25 & 37 & 397 \\
Left frontal & -10 & -24 & 53 & 102 \\
Left supramarginal gyrus & -47 & -49 & 32 & 58 \\
Right middle occipital cortex & -29 & -70 & 14 & 45 \\
\hline
\end{tabular}

Note-All reported statistics are significant at $p<.01$, and all reported clusters contain at least 10 contiguous voxels. There were no regions significantly more active for the opposite comparison.

Although we identified patterns of MPFC activity that correlated with the social and nonsocial nature of the task stimuli, our conclusions may not apply to social cognition directly. In particular, the findings may have resulted from cognitive processes that are required for social cognition but that may not be considered central to social cognition itself. To properly perform in the social task, participants decide whether the stimulus presented is a person or not a person. To properly perform this task, participants must have active a cognitive concept or schema of what a person is and must match that concept or schema to the stimuli before them. If there is a match, the participant can respond person; if there is not a match, the participant can respond not a person.
However, this is a very different type of task than simply indicating valence. Our study indicates that the task context is relevant for identifying social regions of the MPFC, which suggests that social regions may be regions specific to schema matching. Schema matching indeed is a prominent form of social cognition. People seek to make sense of other people, and the use of schemas or mental representations of categories is a useful way to do so (Fiske \& Taylor, 2008). Therefore, neural regions that respond to social cognition may be areas that more broadly respond to matching a stimulus to a mental representation. However, further studies are necessary to dissociate other forms of mental representation matching from social cognition.

Responses within the arMPFC may reflect a multiplicative effect of social information on the processing of valence. This is consistent with the notion that the more anterior and superior areas of the MPFC may calculate more social functions - themselves a type of positive stimuli or value. Presently, the literature makes a ventral-dorsal distinction within the MPFC, asserting that more ventral areas support thinking about the self, whereas more dorsal regions support thinking about others (Mitchell, Macrae, \& Banaji, 2006; Ochsner et al., 2005). In particular, taskdependent MPFC activations during self and other referential thinking suggest that the MPFC mediates metacogni-
A

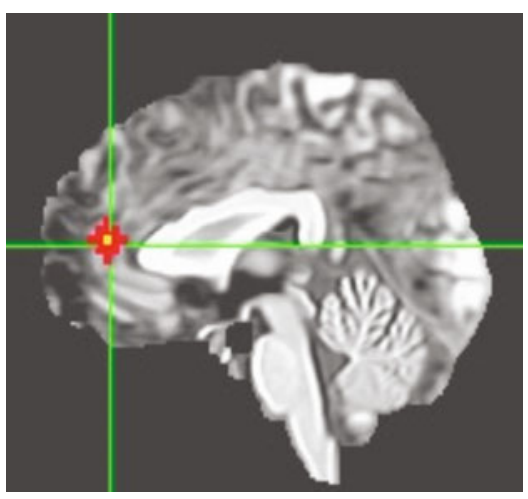

C pACC_Social

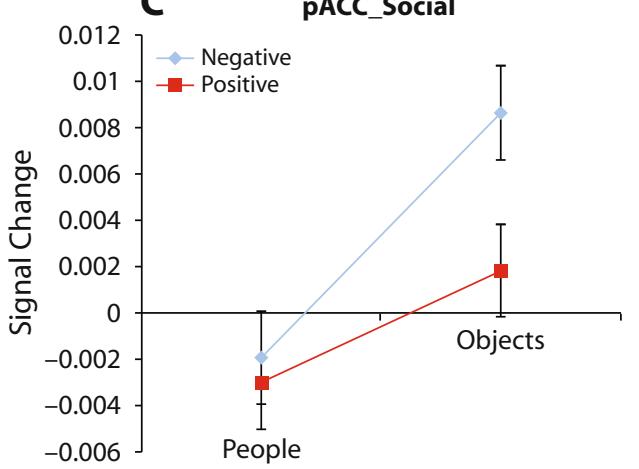

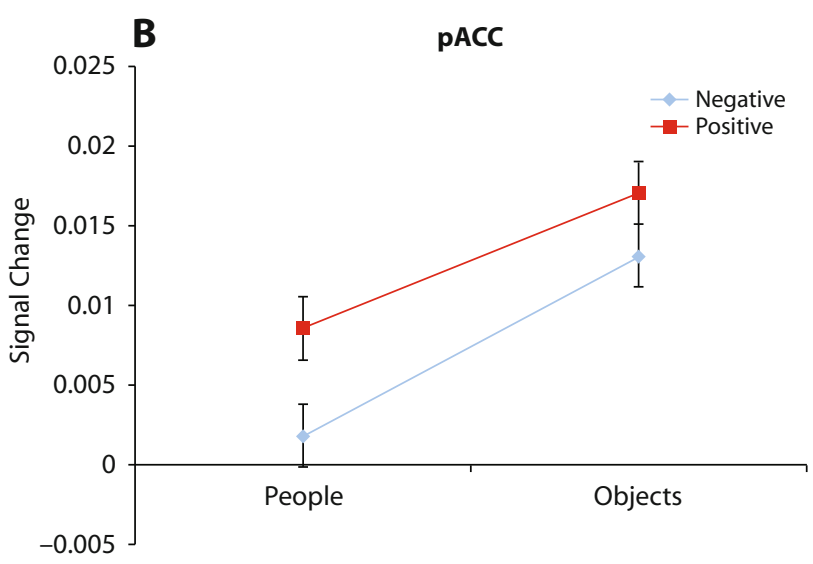

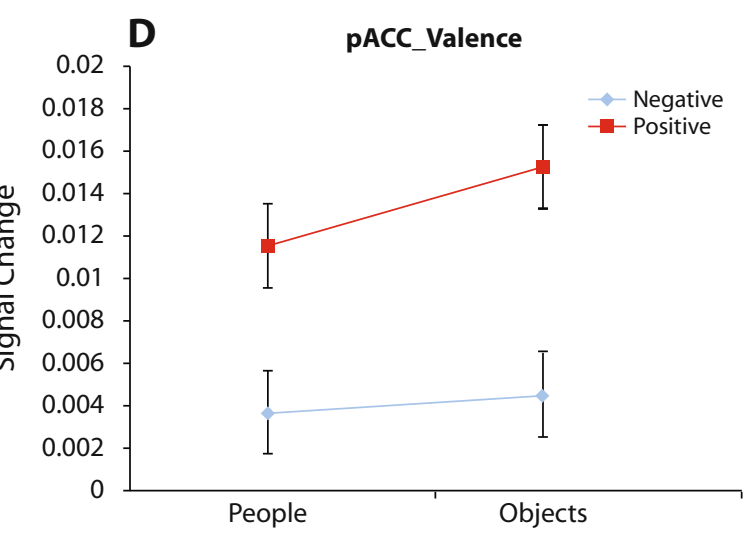

Figure 2. Activations to positive and negative persons and objects in an ROI analysis of the para-anterior cingulate cortex (pACC) within the anterior rostral medial prefrontal cortex. Line graphs correspond to activity in the cluster shaded yellow, although the effect replicates in the larger cluster shaded in red (Talairach coordinates of central voxel: $x=0, y=44, z=12$ ). (A) Cluster of voxels that includes the pACC, located in crosshairs. (B) Main effect. (C) Within social task. (D) Within valance task. 
tive processes recruited for affective evaluation of the self and others (Ochsner et al., 2005). These findings are also consistent with an affective evaluation role of the MPFC.

However, the distinction suggested in the literature does not take into consideration a broader evaluative function of MPFC. As was discussed above, our data support the claim that task demands affect different regions of the MPFC differently. But our findings also suggest that the MPFC may not serve social functions in general and, as such, does not fit with the previous distinctions based simply on social functionality. Nevertheless, the socially tuned activation that we observed within the MPFC are anterior, like those described in the literature that are the basis for the ventral-dorsal distinction. Thus, it may be that the activations observed in these other studies reflect a social evaluative function consistent with what we observed in the present study.

Finally, it is worth noting that the role of the MPFC in affective evaluation fits well with the implication of the MPFC in cognitive control (e.g., Botvinick, Braver, Barch, Carter, \& Cohen, 2001). In particular, recent research by Sugrue, Corrado, and Newsome (2004) has shown that regions of the MPFC integrate reward experience over the recent past. This provides a mechanism for calculating reward rate, which is a critical parameter for monitoring performance. Indeed, recent theories of cognitive control employ reward rate to control the recruitment of executive functions (Aston-Jones \& Cohen, 2005) and the optimization of performance in simple decision-making tasks (Bogacz, Brown, Moehlis, Holmes, \& Cohen, 2006).

\section{AUTHOR NOTE}

L.T.H. is now in the Department of Psychology at New York University; S.M., in the Department of Psychology at Stanford University; W.v.d.B., in the Department of Psychology at Leiden University. Correspondence concerning this article should be addressed to L. T. Harris, Department of Psychology, New York University, Meyer Hall, Room 863, 6 Washington Place, New York, NY 10003 (e-mail: lasana@nyu.edu).

\section{REFERENCES}

Amodio, D. M., \& Frith, C. D. (2006). Meeting of minds: The medial frontal cortex and social cognition. Nature Reviews Neuroscience, 7, 268-277.

Aston-Jones, G., \& Cohen, J. D. (2005). An integrative theory of locus coeruleus-norepinephrine function: Adaptive gain and optimal performance. Annual Review of Neuroscience, 28, 403-450.

Berns, G. S., McClure, S. M., Pagnoni, G., \& Montague, P. R. (2001). Predictability modulates human brain response to reward. Journal of Neuroscience, 21, 2793-2798.

Bogacz, R., Brown, E., Moehlis, J., Holmes, P., \& Cohen, J. D. (2006). The physics of optimal decision making: A formal analysis of models of performance in two-alternative forced choice tasks. Psychological Review, 113, 700-765.

Botvinick, M. M., Braver, T. S., Barch, D. M., Carter, C. S., \& Cohen, J. D. (2001). Conflict monitoring and cognitive control. Psychological Review, 108, 624-652.

Castelli, F., Happé, F., Frith, U., \& Frith, C. (2000). Movement and mind: A functional imaging study of perception and interpretation of complex intentional movement patterns. NeuroImage, 12, 314-325.

Cox, R. W. (1996). AFNI: Software for analysis and visualization of functional magnetic resonance neuroimages. Computers \& Biomedical Research, 29, 162-173.

Daw, N. D., O’Doherty, J. P., Dayan, P., Seymour, B., \& Dolan,
R. J. (2006). Cortical substrates for exploratory decisions in humans. Nature, 441, 876-879.

Delgado, M. R., Miller, M. M., Inati, S., \& Phelps, E. A. (2005). An fMRI study of reward-related probability learning. NeuroImage, 24, 862-873.

FISKE, S. T. (1980). Attention and weight in person perception: The impact of negative and extreme behavior. Journal of Personality \& Social Psychology, 38, 889-906.

FISKE, S. T. (2004). Social beings: A core motives approach to social psychology. New York: Wiley.

Fiske, S. T., CudDY, A. J. C., \& GLICK, P. (2007). Universal dimensions of social perception: Warmth and competence. Trends in Cognitive Sciences, 11, 77-83.

Fiske, S. T., Cuddy, A. J. [C.], Glick, P., \& Xu, J. (2002). A model of (often mixed) stereotype content: Competence and warmth respectively follow from perceived status and competition. Journal of Personality \& Social Psychology, 82, 878-902.

Fiske, S. T., \& TAYLOR, S. E. (2008). Social cognition: From brains to culture. New York: McGraw-Hill.

Fletcher, P. C., Happé, F., Frith, U., Baker, S. C., Dolan, R. J., Frackowiak, R. S. J., \& Frith, C. D. (1995). Other minds in the brain: A functional imaging study of "theory of mind" in story comprehension. Cognition, 57, 109-128.

Forman, S. D., Cohen, J. D., Fitzgerald, M., Eddy, W. F., Mintun, M. A., \& NoLL, D. C. (1995). Improved assessment of significant activation in functional magnetic resonance imaging (fMRI): Use of a cluster-size threshold. Magnetic Resonance in Medicine, 33, 636-647.

Frith, C. D., \& Frith, U. (2006). The neural basis of mentalizing. Neuron, 50, 531-534.

FrITH, U., \& FriTH, C. (2001). The biological basis of social interaction. Current Directions in Psychological Science, 10, 151-155.

Gallagher, H. L., \& Frith, C. D. (2002). Functional imaging of "theory of mind." Trends in Cognitive Sciences, 7, 77-83.

Grèzes, J., Frith, C. D., \& Passingham, R. E. (2004a). Brain mechanisms for inferring deceit in the actions of others. Journal of Neuroscience, 24, 5500-5505.

GrÈzes, J., Frith, C. D., \& Passingham, R. E. (2004b). Inferring false beliefs from the actions of oneself and others: An fMRI study. NeuroImage, 21, 744-750

Hampton, A. N., \& O'Doherty, J. P. (2007). Decoding the neural substrates of reward-related decision making with functional MRI. Proceedings of the National Academy of Sciences, 104, 1377-1382.

Harris, L. T., \& Fiske, S. T. (2006). Dehumanizing the lowest of the low: Neuro-imaging responses to extreme outgroups. Psychological Science, 17, 847-854.

HARRIS, L. T., \& FisKe, S. T. (in press). Brooms in Fantasia: The neural correlates of anthropomorphizing objects. Social Cognition.

Harris, L. T., Todorov, A., \& Fiske, S. T. (2005). Attributions on the brain: Neuro-imaging dispositional inferences beyond theory of mind. NeuroImage, 28, 763-769.

Haxby, J. V., Gobbini, M. I., \& Montgomery, K. (2004). Spatial and temporal distribution of face and object representations in the human brain. In M. Gazzaniga (Ed.), The cognitive neurosciences (pp. 889-904). Cambridge, MA: MIT Press.

Kelley, H. H. (1967). Attribution theory in social psychology. In D. Levine (Ed.), Nebraska Symposium on Motivation (Vol. 15, pp. 192-238). Lincoln: University of Nebraska Press.

Knutson, B., Adams, C. M., Fong, G. W., \& Hommer, D. (2001). Anticipation of increasing monetary reward selectively recruits nucleus accumbens. Journal of Neuroscience, 21(RC159), 1-5.

Knutson, B., Fong, G. W., Bennett, S. M., Adams, C. M., \& HomMER, D. (2003). A region of mesial prefrontal cortex tracks monetarily rewarding outcomes: Characterization with rapid event-related fMRI NeuroImage, 18, 263-272.

Knutson, B., Taylor, J., Kaufman, M., Peterson, R., \& Glover, G. (2005). Distributed neural representation of expected value. Journal of Neuroscience, 25, 4806-4812.

Kwan, V. S. Y., John, O. P., Kenny, D. A., Bond, M. H., \& RobINS, R. W. (2004). Reconceptualizing individual differences in selfenhancement bias: An interpersonal approach. Psychological Review, 111, 94-110. 
Lang, P., \& Bradley, M. (1994). Measuring emotion: The selfassessment manikin and the semantic differential. Journal of Behavior Therapy \& Experimental Psychiatry, 25, 49-59.

Matlin, M. W., \& Stang, D. J. (1978). The Pollyanna principle. Cambridge, MA: Schenkman.

MCArThur, L. A. (1972). The how and what of why: Some determinants and consequences of causal attribution. Journal of Personality \& Social Psychology, 22, 171-193.

McCabe, K., Houser, D., Ryan, L., Smith, V., \& Trouard, T. (2001). A functional imaging study of cooperation in two-person reciprocal exchange. Proceedings of the National Academy of Sciences, $\mathbf{9 8}$, $11832-11835$

McClure, S. M., Ericson, K. M., Laibson, D. I., Loewenstein, G., \& CoHEn, J. D. (2007). Time discounting for primary rewards. Journal of Neuroscience, 27, 5796-5804.

McClure, S. M., Laibson, D. I., Loewenstein, G., \& Cohen, J. D. (2004). Separate neural systems value immediate and delayed monetary rewards. Science, 306, 503-507.

Mitchell, J. P., BanajI, M. R., \& Macrae, C. N. (2005). The link between social cognition and self-referential thought in the medial prefrontal cortex. Journal of Cognitive Neuroscience, 17, 1306-1315.

Mitchell, J. P., Macrae, C. N., \& BanaJi, M. (2006). Dissociable medial prefrontal contributions to judgments of similar and dissimilar others. Neuron, 50, 655 .

Montague, P. R., King-Casas, B., \& Cohen, J. D. (2006). Imaging valuation models in human choice. Annual Review of Neuroscience, 29, 417-448

Nieuwenhuis, S., Slagter, H. A., von Geusau, N., Alting, J., HesLENFELD, D. J., \& HolRoYD, C. B. (2005). Knowing good from bad: Differential activation of human cortical areas by positive and negative outcomes. European Journal of Neuroscience, 21, 3161-3168.

Noveck, I., Goel, V., \& Smith, K. (2004). The neural basis of conditional reasoning with arbitrary content. Cortex, 40, 613-622.

Ochsner, K. N., Beer, J. S., Robertson, E. R., Cooper, J. C., Gabrieli, J. D. E., Kinlstrom, J. F., \& D'Esposito, M. (2005). The neura correlates of direct and reflected self-knowledge. NeuroImage, $\mathbf{2 8}$ 797-814.

O'Doherty, J., Kringelbach, M. L., Rolls, E. T., Hornak, J., \& ANDREWS, C. (2001). Abstract reward and punishment representations in the human orbitofrontal cortex. Nature Neuroscience, 4, 95-102.

O’Doherty, J., Winston, J., Critchley, H., Perrett, D., Burt, D. M., \& Dolan, R. J. (2003). Beauty in a smile: The role of medial orbitofrontal cortex in facial attractiveness. Neuropsychologia, 41, 147-155.

Rilling, J. K., Gutman, D. A., Zeh, T. R., Pagnoni, G., Berns, G. S., $\&$ KILTS, C. D. (2002). A neural basis for social cooperation. Neuron, 35, 395-405

Rilling, J. K., Sanfey, A. G., Aronson, J. A., Nystrom, L. E., \& CoHEN, J. D. (2004). The neural correlates of theory of mind within interpersonal interactions. NeuroImage, 22, 1694-1703.

Sanfey, A. G., Rilling, J. K., Aronson, J. A., Nystrom, L. E., \& CoHEN, J. D. (2003). The neural basis of economic decision-making in the ultimatum game. Science, 300, 1755-1758.

SAXE, R., CAREY, S., \& Kanwisher, N. (2004). Understanding other minds: Linking developmental psychology and functional neuroimaging. Annual Review of Psychology, 55, 87-124.

Saxe, R., \& Wexler, A. (2005). Making sense of another mind: The role of the right temporo-parietal junction. Neuropsychologia, $\mathbf{4 3}$, 1391-1399.

Schultz, W., Dayan, P., \& Montague, P. R. (1997). A neural substrate of prediction and reward. Science, 275, 1593-1599.

Sears, D. O. (1983). The person-positivity bias. Journal of Personality \& Social Psychology, 44, 233-250.

Sugrue, L. P., Corrado, G. C., \& Newsome, W. T. (2004). Neural cor- relates of value in orbitofrontal cortex of the rhesus monkey. Society for Neuroscience Abstracts, 30, 671-678.

Talairach, J., \& Tournoux, P. (1988). Co-planar stereotaxic atlas of the human brain: An approach to medical cerebral imaging. Stuttgart: Thieme.

TAYLOR, S. E., \& BRown, J. D. (1988). Illusion and well-being: A social psychological perspective on mental health. Psychological Bulletin, 103, 193-210.

van den Bos, W., McClure, S. M., Harris, L. T., Fiske, S. T., \& Cohen, J. D. (2007). Dissociating affective evaluation and social cognitive processes in the ventral medial prefrontal cortex. Cognitive, Affective, \& Behavioral Neuroscience, 7, 337-346.

\section{NOTES}

1. We call these pictures positive persons because the valence of all these emotions is either positive (pride) or mixed (both positive and negative; envy, pity). In addition, these emotions are all social; they all require the actual, imagined, or implied presence of another person in order to experience the emotion. This feature of these emotions differentiates them from the emotion elicited by the negative person pictures: disgust - a solely negative emotion that can be elicited without the actual, imagined, or implied presence of another person. Therefore, for the positive emotions, you must mentalize or infer what is in the stimulus target's mind in order to feel pride, envy, or pity. These are secondary or social emotions. You cannot, for instance, be envious of an object without first anthropomorphizing it. However, for the negative pictures, the emotion evoked is the basic emotion disgust, which is not reserved solely for people. Objects can also make you feel disgusted. In addition, valence of the social pictures is on a much shorter range than the range present in the IAPS pictures. The social pictures vary on a range of 3 points on a 7-point Likert scale, whereas the IAPS pictures vary on an entire 9-point Likert scale. Since the highest and lowest rated IAPS pictures were used, it suggests that the IAPS pictures were much more extreme depictions of positive and negative than were the pictures of people. Therefore, the positive and negative distinction between the pictures is a more socially defined positive and negative difference.

2. Although this assertion seems to contradict existing findings in social neuroscience, when the valence and social tasks are separated, there are greater main effects to people than to objects, but just in the social task. This indicates that the task context may be a relevant factor in identifying social regions (see the Discussion section for more on this point).

3. This ROI shares the size and coordinates of the ROI examined in van den Bos, McClure, Harris, Fiske, and Cohen (2007) in this issue. The cluster was based on activations in neural-economic tasks (Knutson et al., 2001; McClure et al., 2004; Nieuwenhuis et al., 2005; O'Doherty et al., 2001; O'Doherty et al., 2003) and social cognition (Gallagher et al., 2002; McCabe et al., 2001; Rilling et al., 2004; Sanfey et al., 2003).

4. The valence $\times$ task interaction is not significant in the pACC. For the social task, the pACC does not differentiate between positive and negative persons. There is more activation to negative objects than to positive objects (see Figure 2C), similar to the pattern observed for the same task in the arMPFC region mentioned above (see Figure 1C). Possibly, the psychological act of negating a category (in this case, the participants' responding not a person to negatively valenced objects) may activate the MPFC. At present, there is no neuroimaging work on the neural basis of negation. But existing work that contrasts modus tollens (if $p$ then $q$, given not $p$, then not $q$ ) with modus ponens (if $p$ then $q$, given $p$ then $q$ ) does find arMPFC activation (Noveck, Goel, \& Smith, 2004).

(Manuscript received February 13, 2007; revision accepted for publication September 26, 2007.) 